\title{
Surfaces
}

\section{L'Infigurable, publié sous la direction de Alexis Nouss, Simon Harel et Michaël La Chance, Montréal, Liber, coll. " Bibliothèque Lilber ", 2000, 195 pages}

\section{Geneviève Sicotte}

Volume 9, 2001

URI : https://id.erudit.org/iderudit/1065069ar

DOI : https://doi.org/10.7202/1065069ar

Aller au sommaire du numéro

Éditeur(s)

Les Presses de l’Université de Montréal

ISSN

1188-2492 (imprimé)

1200-5320 (numérique)

Découvrir la revue

Citer ce compte rendu

Sicotte, G. (2001). Compte rendu de [L'Infigurable, publié sous la direction de Alexis Nouss, Simon Harel et Michaël La Chance, Montréal, Liber, coll.

"Bibliothèque Liber ", 2000, 195 pages]. Surfaces, 9.

https://doi.org/10.7202/1065069ar 


\section{Nouss, Harel et La Chance - L'Infigurable}

Geneviève Sicotte

Collège André-Grasset

Surfaces vol IX 1.01 (v.1.0F - 15/12/2001) - ISSN:

1188-2492

Tout texte reste la propriété de son auteur. Néanmoins, SURFACES demande d'être citée à l'occasion de toute autre publication du texte en question.

\section{L'Infigurable, publié sous la direction de Alexis Nouss, Simon Harel et Michaël La Chance, Montréal, Liber, coll. "Bibliothèque Liber", 2000, 195 pages.}

C'est une expérience qu'ont fait tous ceux qui vivent pour et dans le langage. Soudain, dans l'écriture ou le discours, les mots nous échappent, nous manquent, il y a du flou, le verbe s'avère déficient et insuffisant; et pourtant, au moment même où l'on constate cette déficience et cette insuffisance, on sait bien que le verbe est notre seule ressource, et qu'il faut continuer. Le langage indique ses limites mais désigne par là autre chose dont il est aussi porteur, fait surgir du sens dans la faille et le manque. Traiter de cette part mystérieuse qui, passant par le langage, l'excède néanmoins, c'est le projet que se sont donnés les collaborateurs réunis par Alexis Nouss, Simon Harel et Michaël La Chance dans l'ouvrage recensé ici. Puisque l'infigurable - pour reprendre le vocable choisi par les auteurs - ne saurait se laisser réduire ou expliquer, qu'il est ce qui échappe à toute mise en forme, un discours théorique sur la question peut sembler entaché dès le départ d'un vice de forme. Il n'en reste pas moins que selon les auteurs, 
quelque chose de l'infigurable peut être exposé, montré, et compris. Démarche exigeante et inévitablement paradoxale, puisqu'elle conduira à théoriser ce qui se refuse à être théorisé, à mettre en mots et en images ce qui échappe au discours, au sens, à la mise en forme. Mais démarche d'autant plus audacieuse, car elle embrasse le paradoxe qui la fonde plutôt que de tenter de s'y soustraire. Pour les auteurs de ce collectif, l'infigurable, la présence essentielle d'un non-dit au cœur du dit, plutôt que de provoquer le silence, est ce qui amène le discours, dans une tentative de faire jaillir le sens. À cet égard, l'un des intérêts de ce livre est sans aucun doute qu'il rassemble non seulement des textes théoriques, mais aussi de courtes fictions, des dessins, des photos, bref de quoi tenter d'approcher, selon ses multiples facettes, un objet complexe.

On trouvera sans doute, au fil des textes rassemblés, diverses définitions de ce qu'est l'infigurable, d'autant plus que la notion est abordée à travers les prismes variés que sont l'ethnologie, la psychanalyse, les études littéraires ou l'esthétique. Néanmoins, l'espace balisé par le recueil est traversé par des convergences certaines. L'infigurable, c'est ce qui ne peut se dire, non parce que cela serait irreprésentable, mais parce que le dire, qui est toujours porteur de sens, en dénaturerait l'infigurabilité. L'infigurable néanmoins existe, comme une sorte de rémanence rétinienne, image fantôme qui, en surimpression sur l'œuvre, lui confère son épaisseur, sa dimension irréductible - absence qui donne présence, sans que cela soit jamais réparation ou rédemption de l'absence. On conçoit dès lors que la réflexion sur l'infigurable soit foncièrement liée à des expériences de la limite, qui sapent les fondements mêmes du sens : l'identité et l'altérité, la folie, la mort et plus que tout l'Holocauste, qui devient en quelque sorte le centre de gravité - centre de gravité en forme de trou noir - de cette réflexion.

L'un des articles les plus intéressants du recueil est celui d'Alexis Nouss qui, à l'occasion d'une lecture de la poésie de Paul Celan, offre généreusement au lecteur des pistes pour mieux approcher la notion d'infigurable. Pour Nouss, la poésie de Celan est traversée de manière fondatrice par le paradoxe de l'infigurable, puisqu'elle emploie le langage comme " seul possible qui puisse dire l'impossible du réel " (p. 140). Il poursuit : " La fonction du récit de l'indicible n'est plus tant de raconter que de servir de médium à l'indicible " (p. 140). Il y aurait donc, dans tout langage, quelque chose qui se montre sans être exprimé, une part obscure qui, échappant au langage, serait quand même évoquée - ou invoquée - 
par lui. Cette part, pour Nouss, serait l'infigurable, point de fuite de la poésie de Celan, mais aussi plus généralement de la littérature de la Shoah. Pourtant, selon Nouss, l'infigurable ne doit pas être réduit à l'irreprésentable ou à l'indicible : il faut du verbe pour que surgisse l'important, qui est bien dans ce qui ne peut être raconté, mais reste en creux dans le verbe. Cette explication permet de mieux comprendre pourquoi Nouss place une certaine conception du témoignage au cœur de sa démarche, conception qu'il redéfinit par rapport à celles de Primo Levi et de Lyotard. Ces deux derniers effectuent une critique radicale du témoignage - témoigner de la Shoah, c'est trahir, clôre un chapitre, donner à entendre que ce qui a eu lieu est terminé et peut désormais se raconter; d'où le fait que le témoignage ainsi conçu " efface par sa possibilité ce qu'il entend transmettre " (p. 136). Mais Nouss tente de redéfinir le témoignage. Pour lui, " la vibration chez Rothko ", " l'espace vide si angoissant [...] dans les toiles de De Chirico ou de Magritte ", " le silence chez John Cage, la syncope atonale de Schoenberg, et dans le domaine littéraire, les poèmes de Celan ou l'œuvre de Beckett " (p. 143) exposent non le sens mais le vide, montrent ce qui ne peut se montrer, placent l'infigurable au cœur de la relation qui unit le récepteur et l'œuvre. C'est ainsi qu'elles témoignent, et qu'elles font aussi du récepteur un témoin. Contrairement à certains qui prétendent que toute parole ou tout témoignage sur la Shoah est d'emblée non valide et même violent et " exterminateur ", Nouss croit que l'infigurable se montre à travers l'art, mais qu'il doit, pour être fidèle, témoigner d'une perte, d'une absence.

Ellen Corin aborde la question de l'infigurable par un biais différent, celui de son travail en tant qu'anthropologue et psychanalyste. Revenant en particulier sur des rituels de possession par les esprits qu'elle a étudiés au Congo, elle explore la " figure de l'étranger " (p. 23) en soi et en dehors de soi, c'est-à-dire la possession en tant que phénomène de l'étranger en soi - l'esprit dont sont habitées les possédées - , et le contact de l'ethnographe avec cette étrangeté. C'est un parcours personnel qu'elle propose, balisé par des décentrements liés à sa compréhension changeante des phénomènes qu'elle a tenté d'analyser. Elle évoque avec beaucoup de sensibilité les transes des possédées, surtout des femmes, qui mettent en place des pratiques symboliques radicales où se manifeste le " pouvoir déstabilisant des marges par rapport aux figurations centrales qu'une société se donne d'elle-même " (p. 26). Mais pour Corin, cette explication, que ne récuserait pas l'analyse anthropologique classique étudiant le code et 
ses mises en tension, ne dit pas tout et élude l'infigurabilité de ces pratiques. Il y a dans les transes, dans certains regards de possédées en particulier, un surplus de signifiance que l'anthropologue ne peut saisir, qui échappe à la figuration de la discipline scientifique. C'est à ce surplus qu'elle s'arrête, à cet impensé " qui toujours demeure en excès de la représentation que je peux m'en faire " (p. 28), et que l'anthropologie trahit jusqu'à un certain point en le reconfigurant, en le faisant entrer dans un cadre analytique. Il y a donc dans l'expérience décrite par Corin un double décentrement - le premier naissant du choc culturel, du " brouillage des frontières " (p. 33), et dont la mise en discours constitue l'objet propre de l'anthropologie, et le second provoqué par l'écho empathique qu'éveille en elle l'expérience de la possession où, à la faveur d'un regard posé sur elle par une possédée, " le contact avec l'autre étranger fraie au-dedans de nous un passage, ouvre une échancrure par où le sauvage, le non-familier peuvent faire surface " (p. 33). Tout le souci de Corin consiste à ne pas dissocier ces deux axes en préservant la possibilité d'une parole. De là le lien avec la psychanalyse, autre versant de sa pratique qui place aussi en son centre le langage tel qu'il est toujours à la fois porteur de sens et marqué par " un sens qui ne se dit que de biais, d'une manière allusive, infiniment déplacée " (p. 37).

De nombreux autres textes mériteraient qu'on s'y arrête aussi longuement. Mais puisqu'il ne s'agit pas ici de résumer en détail l'ensemble du recueil, je mentionnerai simplement quelques thèmes qui pourront orienter le lecteur dans son parcours. Dans un article exigeant qui retiendra surtout les spécialistes, Simon Harel examine l'infigurable comme lieu privilégié de la psychanalyse. Cet infigurable fait surface particulièrement dans la parole psychanalytique, puisque celle-ci est toujours le lieu d'une confusion qui est aussi " la condition même de la symbolisation " (p. 48). De la confusion à la symbolisation, du dessaisissement au ressaisissement (et vice-versa), le processus psychanalytique se constituerait ainsi sur le désir de signifier l'infigurable - de le signifier, et non de le figurer. Dès lors, lorsque l'analysant acquiert le " don des langues " (p. 48) c'est-à-dire lorsqu'il accède à une parole significative il n'entre pas dans la maîtrise du sens, mais plutôt dans une " relation d'inconnu " (p. 56) face au sens et à luimême. Alain Médam explore pour sa part les liens entre l'infigurable et l'esthétique moderne. L'évolution de l'art au XXe siècle, et en particulier l'abandon de la figuration, lui semble orientée par une tentative de faire droit à l'infigurable. Son article aboutit 
malheureusement à une opposition trop simple entre la science, qui serait " prisonnière de l'ordre du trait, de la direction linéaire ", et l'art contemporain, " qui nous permettrait de saisir la complexité, l'équivocité, l'incommensurabilité, l'infigurabilité des objets du présent " (p. 97). Gabriel Louis Moyal aborde dans une réflexion croisée trois ordres de signification : les paradigmes (au sens kuhnien), le corps et l'écrit. Ces ordres de signification sont si massifs qu'ils en deviennent invisibles, tellement présents qu'on ne les voit plus. Et pourtant, l'analyse les montre éclatés, morcelés : le paradigme se révèle comme figure parmi d'autres du discours, le corps se fétichise et ses gestes deviennent des signes ininterprétables, le texte n'est jamais certain, donne lieu à des exégèses contradictoires. Rien n'est fondateur. Pierre Ouellet propose une lecture de Rothko qui prend appui sur des textes du peintre lui-même, et fait voir que celui-ci conçoit la peinture comme trace visible d'un infigurable. Pour Rothko, la peinture contemporaine ne peut être fondée sur le percept, car ce qui se montre en elle " se refus[e] à la monstration " (p. 159). Elle n'a de valeur au sens éthique du terme - que si elle témoigne d'un affect, d'un " état pathique ou passionnel qui est un ethos au sens propre, une pré-disposition en soi infigurable, irreprésentable" (p. 161). L'œuvre d'art " vise un manque qu'aucune figure ne peut combler, à la place de quoi l'affect lui-même laissera une trace, tableau vivant d'une incommunicable angoisse, d'une infigurable soif, qui fait qu'on communique tous ensemble par le même manque, qu'on se représente tous dans la même solitude, enfin partagée " (p. 163). Le recueil se clôt sur un entretien de Michaël La Chance avec Ariane Thézé, dont quelques œuvres sont reproduites au fil des textes. L'artiste explique la position singulière qu'elle adopte dans son travail, et qui fait qu'étant simultanément derrière et devant la caméra, elle occupe une position dédoublée et floue acteur et auteur, sujet représenté et représentant. Ce faisant, elle tente de donner à voir un personnage " à la fois absent et présent " (p. 190), en proie à des déformations et à des dissolutions qui permettent l'émergence d'un infigurable du sujet et de l'identité.

Semés au fil du recueil, plusieurs textes de création viennent jeter un éclairage différent sur la notion d'infigurable et complètent en quelque sorte des textes théoriques inévitablement plus distanciés face à leur objet. Dans une courte fiction qui désamorce sans cesse tout effet de véridicité du récit, David Albahari aborde le thème des rapports entre l'écriture et la mort. Ruben Rubenovic, un ancien marchand de tissu décrit de 
manière ironique comme le " soi-disant prototype du Juif " (p. 16), agonise dans la sérénité, " blême, paisible, somptueux " (p. 12). À ses côtés, les parents du narrateur, l'assistant anxieusement dans ces heures ultimes, ainsi que deux enfants, une petite fille et un petit garçon - le double du narrateur. Comment faire du récit avec la mort ?, semble demander l'écrivain. Au nom de quoi, de quelle souffrance, de quelle arrogance ou de quel désir irrationnel de se faire entendre, fouiller " comme des vautours le cimetière de papier " ? (p. 11) Mais malgré tout le récit est là, existant de toutes ces hésitations, ces doutes et ces remises en question, et laissant finalement en partage au lecteur quelque chose de l'expérience de la mort, qui semble ici reliée de manière vitale à l'expérience de l'écriture elle-même. Michaël La Chance signe pour sa part un émouvant essai poétique qui constitue un des sommets du recueil. Il y traite de l'infigurable qui surgit dans l'expérience de la perte - perte évoquée ici à demi-mots, liée à la mort, au suicide peut-être, d'un être aimé : " Est-ce à perdre, perdre encore, que nous apprenons ce qu'il y a de plus précieux? Nos pertes révèlent la nature du monde comme Énigme, elles permettent à une vérité du monde de se connaître et de s'accomplir dans un arrachement dont nous sommes la mesure " (p. 67). La perte dévaste, creuse " un trou dans le langage " (p. 65), mais malgré tout, l'être garde un recours, celui même du langage troué, de l' "interrogation de la vie " (p. 65) : " Il me reste, / pour me faire exister / À convoquer / ce qui prend refuge dans la voix " (p. 64). Catherine Mavrikakis aborde elle aussi la mort et le suicide, mais dans un récit d'une tout autre facture, violent, énigmatique, où se brouillent à la fois la temporalité et l'identité. Peter Nádas propose un dialogue aux accents beckettiens qui, d'un ton distancié et bouffon, fait se croiser les thèmes de la vacuité de la parole, de l'absence de communication, de la peur et de l'échec. Enfin, dans une nouvelle en forme d'hommage à Walter Benjamin, Régine Robin reconfigure un thème essentiel de l'auteur des Passages, celui du nécessaire dévoilement de l'ancien ici, l'ancien comme infigurable - dans le présent. Elle met en scène une " chiffonnière du temps " (p. 184) engagée dans une quête identitaire problématique et qui, établie dans une Berlin contemporaine recouverte par les simulacres du présent, exhume des lambeaux du passé, tente de faire parler ces " traces de traces " ( $p$. 184). À ces textes s'ajoutent des photographies et dessins d'Alain Médam, James Nachtwey, L. Somlósi, Ariane Thézé et Denis Pellerin qui offrent prise à la rêverie du lecteur et continuent dans l'image ce que le texte a commencé. 
Cet ouvrage intéressera évidemment les chercheurs qui étudient la Shoah, la mort, la folie, les décentrements identitaires, les expériences de la limite, mais aussi les adeptes d'une critique d'inspiration psychanalytique pratiquée sur le terrain de l'anthropologie, de l'analyse littéraire ou de l'esthétique. Pour autant, il n'est pas réservé qu'à ces seuls spécialistes, et les chercheurs en analyse littéraire y trouveront l'occasion d'une réflexion stimulante sur la notion de représentation. C'est du moins l'aspect qui, en tant que chercheuse en sociocritique, m'a le plus retenue. En effet, la sociocritique actuelle se présente le plus souvent comme l'étude des représentations : représentations des savoirs, des usages sociaux, des configurations symboliques et idéologiques. Or la perspective introduite par l'ouvrage déplace radicalement les termes du débat, puisqu'elle questionne la pertinence même de la notion de représentation. L'important n'est pas dans ce qui est représenté, mais bien dans ce qui ne l'est pas, ou dans ce qui passe à côté, dans les marges, entre les lignes de la représentation. On peut évidemment récuser cette vision pour se réclamer d'une approche pragmatique : la représentation discursive de tel sujet, c'est ce qui se dit et s'écrit sur ce sujet. Et l'on n'aurait certes pas tort, puisque le caractère opératoire de cette manière d'envisager les choses est jusqu'à un certain point la preuve de sa validité - puisque les chercheurs réussissent bien à mettre le doigt sur quelque chose et à faire émerger du sens caché de ces représentations. Mais une telle approche comporte aussi un danger, celui de reconduire une conception transparente de la signification dont il faut admettre qu'elle ne tient pas face aux avancées de la sémiotique ou de la philosophie du langage. Les chercheurs en sociocritique pourraient par conséquent valoriser un concept dynamique de représentation qui ne soit pas uniquement fondé sur la positivité du langage, mais qui donne voix à l'opacité, aux silences, aux déplacements. $\mathrm{Si}$, comme le dit Ellen Corin, toute figuration " ne se soutient que de son rapport à l'infigurable " (p. 22), cela invite à envisager la représentation par rapport à tout ce qu'elle tait, à tout ce qu'elle ne représente pas, à tout un envers du dire qui détermine ce qui est dit.

$\mathrm{Au}$ terme de ce recueil, il me reste malgré tout un regret, ou plutôt le sentiment d'une incomplétude, inévitable sans doute, mais qui pourrait appeler d'autres travaux sur le même sujet. L'Holocauste, la folie ou la mort sont des expériences extrêmes, et leur caractère tragique n'est sans doute nulle part mieux manifesté que dans cet espace d'infigurabilité exploré ici : " Nous cherchons la réponse précisément là où la douleur s'est 
chevillée - car c'est là qu'est préservée une interrogation de la vie " (p. 65), dit Michaël La Chance. Mais ce parti-pris de douleur confère au recueil une tonalité bien noire. Sans doute, les auteurs ne font qu'analyser le matériau qu'ils ont sous la main : pour des raisons sociologiques et historiques, la modernité du XXe siècle s'est bâtie sur un ethos tragique auquel les événements ont bien souvent fait écho ou qu'ils ont relancé. Étudier l'art moderne, c'est affronter la perte du sens, le désespoir, la mort. Ce n'est pourtant pas une raison pour se limiter à cet aspect de l'existence. Il semblerait à cet égard fructueux d'explorer ce que j'appellerai après Simon Harel des " figures heureuses de l'infigurable " (p. 48), qui existent dans de nombreuses œuvres et s'expriment à travers des objets qui sont tout aussi constitutifs de l'expérience humaine : l'amour, l'extase, l'enfantement, le jeu pur, le sentiment cosmique. N'y a-t-il pas, là aussi, dissolution de l'individualité, de la parole, du sens, et malgré tout possibilité de témoigner de cette dissolution ? Bien sûr, il y a toujours ce danger : la joie peut être vue comme rédemption, et l'œuvre nierait alors, dans une fausse positivité, et l'infigurable, et le tragique. Mais une exploration critique comparable à celle menée dans le présent recueil éclairerait justement cette question dans toute sa complexité.

Accueil Surfaces | Table des matières | Recherche Surfaces Home Page | Table of Contents | Search

PUM | Livres | Revues | Publications électroniques | Vente et distribution 